\title{
INDUCTIVE DEFINITIONS, MODELS OF COMPREHENSION AND INVARIANT DEFINABILITY ${ }^{\dagger}$
}

\author{
BY
}

K. R. APT

ABSTRACT

\begin{abstract}
The connections between inductive definability and models of comprehension are studied. Let $U=\left\langle A, R_{1}, \cdots, R_{n}\right\rangle$ be an infinite structure and let $I_{\phi}$ be a set inductively defined by a formula $\phi$ of the second order language $L_{2}$. We prove that if $\mathscr{A}$ is a model of $\Delta_{1}$-Comprehension relativized to $\phi$, and $\phi$ is $\mathscr{A}$-absolute, then for every $\eta$ smaller than the height of $\mathscr{A}(h(\mathscr{A})), I_{\phi}^{n}$ is in $\mathscr{A}$. If $\mathscr{A}$ is a $\beta$-structure which satisfies $\Sigma_{1}$-Comprehension relativized to $\phi$ and WF $(X)$, and $\phi$ is $\mathscr{A}$-absolute, then $I_{\phi}$ is in $\mathscr{A}$ and $\| \phi \mid<h(\mathscr{A})$. These results imply that Barwise-Grilliot theorem is false in the case of uncountable acceptable structures. We also study the notion of invariant definability over models, of $\Delta$-Comprehension.
\end{abstract}

\section{$\S 1$. Introduction}

This paper is devoted to the study of connections between inductive definability and models of comprehension. The basic question to which we want to find an answer is the following.

Let $\mathcal{U}$ be an infinite structure. Suppose that $I_{\phi}$ is a set inductively defined by a second order formula $\phi$. Which properties of a second order structure $\mathscr{A}$ over $\mathcal{U}$ imply that $I_{\phi}$ or some of its stages $I_{\phi}^{\eta}$ belong to $|\mathscr{A}|$ ?

We prove in $\S 3$ that if $\mathscr{A}$ satisfies $\Delta_{1}^{1}$-Comprehension scheme relativized to $\phi$ and $\phi$ is $\mathscr{A}$-absolute then for every $\eta$ smaller than the height of $\mathscr{A}(h(\mathscr{A}))$ $I_{\phi}^{\eta} \in|\mathscr{A}|$.

If we assume that $\mathscr{A}$ is a $\beta$-structure for which $\phi$ is $\mathscr{A}$-absolute and $\mathscr{A}$ satisfies $\Sigma_{1}$-Comprehension relativized to $\phi$ and WF $(X)$ then $\|\phi\|<h(\mathscr{A})$ and $I_{\phi} \in|\mathscr{A}|$.

Using these results we prove that Barwise-Grilliot theorem (see Moschovakis [2] p. 140) is false in the case of uncountable acceptable structures.

+ This paper is registered as Report ZW 69/76 of the Mathematical Centre.

Received April 29, 1976 
The basic problem to which we are unable to find the answer is the following: is it true that hyperelementary relations on an arbitrary infinite structure $\mathcal{u}$ form a model (the smallest one) of $\Delta_{1}^{1}$-Comprehension?

In $\$ 4$ we study the notion of invariant definability over models of $\Delta_{1}^{1}$ Comprehension. We prove that if $\mathcal{U}$ is a countable acceptable structure and $T$ is an inductive theory in $L_{2}^{u}$ which extends $\Delta_{1}^{1}$-Comprehension and has a model over $\mathcal{U}$ then the hyperelementary relations of $\mathcal{U}$ are exactly the sets invariantly ddefinable over models of $T$. This theorem is not true in the case of uncountable acceptable structures.

Finally we prove that if $\mathcal{U}$ is acceptable then for some inductive set $I$ every set invariantly definable over models of $\Delta_{1}^{1}$-Comprehension is hyperelementary in $I$.

Unfortunately we are unable to give the exact characterization of sets which are invariantly definable over all models of $\Delta_{1}$-Comprehension in the case of an arbitrary infinite structure $\mathcal{U}$.

We thank Mr. E. Alward and Prof. G. Kreisel for helpful remarks about $\Delta \mathrm{i}$-Comprehension.

\section{§2. Preliminaries}

Throughout the paper the letters $\eta, \xi, \tau, \sigma$ always denote ordinals. If $A$ is a set we use small latin letters to denote the elements of $A$ and capital letters to denote relations on $\mathrm{A}$ of any (finite) number of arguments. By $A^{n}(n \geqq 1)$ we denote the set of all $n$-tuples of elements of $A$. If $X$ is a set $\mathscr{P}(X)$ denotes its power set. $\bar{x}$ denotes a sequence $x_{1}, \cdots, x_{n}$ of elements of $A$ and $\bar{X}$ denotes a sequence $X_{1}, \cdots, X_{n}$ of relations on $A$.

For the convenience of the reader we recall here some definitions and notations which can be found in Moschovakis [2] and Moschovakis [3].

The first order language over a set $A, L^{A}$ has an infinite list of individual variables $x, y, z, \cdots$, a constant $b$ for each element $b$ of $A$, an infinite list $X, Y, Z, \cdots$ of $n$-ary relation variables for each $n \geqq 1$ and a constant $P$ for each relation $P$ on $A$. In forming formulas of $L^{A}$ the quantifiers $\exists$ and $\forall$ are applied only to individual variables.

The second order language over $A, L_{2}^{A}$ is obtained by allowing quantification of the relation variables in the language $L^{A}$. For convenience we assume that the formulas of the type $X=Y$ are not well formed formulas (we may write instead $\forall x(X(x) \leftrightarrow Y(x)))$

Let $\mathcal{U}=\left\langle A, R_{1}, \cdots, R_{n}\right\rangle$ be a structure (that is to say $R_{1}, \cdots, R_{n}$ are relations over $A$ ). The first (second) order language $L^{q u}\left(L_{2}^{q}\right)$ for $U$ consists of those 
formulas of the language $L^{A}\left(L_{2}^{A}\right)$ whose relation constants are among $=, R_{1}, \cdots, R_{n}$. We sometimes write $\bar{x} \in X$ instead of $X(\bar{x})$.

Formulas of $L^{u}$ are called arithmetical formulas. All structures considered are infinite.

$\mathscr{A}$ is called a second order structure over $\mathscr{U}=\left\langle A, R_{1}, \cdots, R_{n}\right\rangle$ if $\mathscr{A}=$ $\left\langle A,|\mathscr{A}|, R_{1}, \cdots, R_{n}\right\rangle$ where $|\mathscr{A}| \subset \cup_{n \geqq 1} \mathscr{P}\left(A^{n}\right)$. While interpreting a formula $\phi$ of $L_{2}^{\mathscr{Q}}$ on $\mathscr{A}$ we assume that the second order quantifiers of $\phi$ range over $|\mathscr{A}|$. For simplicity $\mathscr{P}(\mathcal{U})$ denotes the second order structure $\left\langle A, \bigcup_{n \geqq 1} \mathscr{P}\left(A^{n}\right), R_{1}, \cdots, R_{n}\right\rangle$.

We sometimes write $\phi(\bar{x}, \bar{X})$ instead of $\mathscr{P}(\mathscr{U}) \vDash \phi(\bar{x}, \bar{X})$.

Definition 1. Let $\mathscr{A}$ be a second order structure over $\boldsymbol{u}$. A formula $\phi\left(x_{1}, \cdots, x_{k}, X_{1}, \cdots, X_{n}\right)$ of $L_{2}^{\mu}$ with free variables indicated is called $\mathscr{A}$-absolute if

$$
A \vDash \phi\left[x_{1}, \cdots, x_{k}, X_{1}, \cdots, X_{n}\right] \Leftrightarrow \mathscr{P}(\mathcal{U}) \vDash \phi\left[x_{1}, \cdots, x_{n}, X_{1}, \cdots, X_{n}\right]
$$

for all $x_{1}, \cdots, x_{k} \in A$ and $X_{1}, \cdots, X_{n} \in|\mathscr{A}|$.

$\mathrm{WF}(X)$ is the following formula of $L_{2}^{\mathscr{q}}$ where $U=\left\langle A, R_{1}, \cdots, R_{n}\right\rangle$ : $\mathrm{WF}(X) \leftrightarrow X$ is a well-founded transitive relation on $A$, i.e.

$$
\begin{aligned}
\mathrm{WF}(X) \leftrightarrow & \forall x, y, z[((x, y) \in X \wedge(y, z) \in X) \rightarrow(x, z) \in X] \\
& \wedge \forall S[\exists x(x \in S) \rightarrow \exists y(y \in S \wedge \forall x(x \in S \rightarrow(x, y) \notin X))]
\end{aligned}
$$

Definition 2. A second order structure $\mathscr{A}$ over $U$ is called a $\beta$-structure if the formula $\mathrm{WF}(X)$ is $\mathscr{A}$-absolute.

If $\phi\left(x_{1}, \cdots, x_{k}, X_{1}, \cdots, X_{n}\right)$ is a formula of $L_{2}^{q_{l}}$ with free variables indicated we say that $\phi$ is a $\left(k, r_{1}, \cdots, r_{n}\right)$-formula to indicate the fact that $\phi$ has exactly $k$ free individual variables and for every $i \leqq n$ free variable $X_{i}$ ranges over $r_{i}$-ary relations.

Definition 3. Let $\phi(\bar{x}, X, \bar{X})$ be a $\left(m, l, r_{1}, \cdots, r_{n}\right)$-formula of $L_{2}^{q_{l}}$ and $\psi\left(\bar{y}, \bar{y}_{1}, \bar{Y}\right)$ be a $\left(l+p, p_{1}, \cdots, p_{r}\right)$-formula of $L_{2}^{q}$. Suppose that $\phi(\bar{x}, X, \bar{X})$ and $\psi\left(\bar{y}, \bar{y}_{1}, \bar{Y}\right)$ have no variables in common. Then $\phi_{\bar{y}}\left(\bar{x}, \psi\left(\bar{y}, \bar{y}_{1}, \bar{Y}\right), \bar{X}\right)$ is a $\left(m+p, r_{1}, \cdots, r_{n}, p_{1}, \cdots, p_{r}\right)$-formula of $L_{2}^{q_{1}}$ obtained from $\phi(\bar{x}, X, \bar{X})$ by replacing all the atomic formulas of the form $\bar{z} \in X$ occurring in $\phi(\bar{x}, X, \bar{X})$ by $\psi\left(\bar{z}, \bar{y}_{1}, \bar{Y}\right)$.

Lemma 1. Suppose that $\phi(\bar{x}, X, \bar{X})$ and $\psi\left(\bar{y}, \bar{y}_{1}, \bar{Y}\right)$ are formulas of $L_{2}^{q_{2}}$. which satisfy the above conditions. Then for all $\bar{x}, \bar{y}_{1}, \bar{X}$ and $\bar{Y}$ 
where

$$
\mathscr{P}(U) \vDash \phi_{\bar{y}}\left(\bar{x}, \psi\left(\bar{y}, \bar{y}_{1}, Y\right), \bar{X}\right) \Leftrightarrow \mathscr{P}(U) \vDash \phi(\bar{x}, A, \bar{X})
$$

$$
A=\left\{\bar{y}: \mathscr{P}(\mathcal{U}) \vDash \psi\left(\bar{y}, \bar{y}_{1}, \bar{Y}\right)\right\}
$$

Proof. By induction on the length of the formula $\phi(\bar{x}, X, \bar{X})$.

Definition 4. Suppose that $X \subset A^{2}$ is a binary relation on $A$. Let

$$
\operatorname{Fld}(X)=\{x: \exists y((x, y) \in X \vee(y, x) \in X)\}
$$

If $z \in \operatorname{Fld}(X)$ then

$$
X \uparrow_{z}=\{(x, y):(x, y) \in X \wedge(y, z) \in X\} .
$$

By transfinite induction we define the classes of well-founded transitive relations on $A$

$$
\begin{aligned}
\mathrm{WF}(\sigma)= & \{X: X \text { is a well-founded transitive relation on } A \text { and } \\
& \left.\forall z\left(z \in \mathrm{Fld}(X) \rightarrow X \uparrow_{z} \in \mathrm{WF}(\tau) \text { for some } \tau<\sigma\right)\right\} .
\end{aligned}
$$

It is easy to see then that

$$
\mathrm{WF}(X) \Leftrightarrow X \in \mathrm{WF}(\sigma) \text { for some } \sigma .
$$

Suppose that WF $(X)$. By $\|X\|$ we denote the least $\sigma$ such that $X \in \mathrm{WF}(\sigma)$. If $x \in \operatorname{Fld}(X)$ then $\left\|X \uparrow_{x}\right\|<\|X\|$. If $(x, y) \in X$ then $X \uparrow_{x}=X \uparrow_{y} \uparrow_{x}$. If $\sigma<\|X\|$ then for some $x \in \operatorname{Fld}(X) \sigma=\left\|X \uparrow_{x}\right\|$.

If $X$ and $Y$ are two binary relations on $A$ then by $X \leqq Y$ we mean the following formula of $L_{2}^{A}$ :

$\exists Z(Z$ is a $1-1$ function from $\operatorname{Fld}(X)$ into $\operatorname{Fld}(Y)$ and $\forall x, y((x, y) \in X \rightarrow(Z(x), Z(y)) \in Y))$.

We define then

$$
X<Y \leftrightarrow \exists z\left(z \in \operatorname{Fld}(Y) \wedge X \leqq Y \uparrow_{z}\right)
$$

It is easy to see then that if $\operatorname{WF}(Y)$ then

$$
\begin{aligned}
& X \leqq Y \Rightarrow\|X\| \leqq\|Y\|, \\
& X<Y \Rightarrow\|X\|<\|Y\| .
\end{aligned}
$$

Definition 5. Let $\mathscr{A}$ be a second order structure over $\mathcal{u}$. We define

$$
h(\mathscr{A})=\sup (\|X\|+1: \mathscr{P}(\mathcal{U}) \vDash \mathrm{WF}(X) \text { and } X \in|\mathscr{A}|) \text {. }
$$

We call $h(\mathscr{A})$ the height of $\mathscr{A}$. 
Definition 6. Let $\mathscr{K}$ be a class of structures over $\mathcal{U}=\left\langle A, R_{1}, \cdots, R_{n}\right\rangle$. Then

$\cap \mathscr{K}=\{X: X \in|\mathscr{A}|$ for all $\mathscr{A} \in \mathscr{K}\}$

$$
\begin{aligned}
\operatorname{Def}(\mathscr{K})=\{ & X: X \in \cup_{n \geqq 1} \mathscr{P}\left(A^{n}\right) \text { and for some formula } \phi(\bar{x}) \text { of } L_{2}^{\mathscr{U}} \\
& \text { with free variables indicated which is } \mathscr{A} \text {-absolute } \\
& \text { for all } \mathscr{A} \in \mathscr{K} \\
& \mathscr{P}(\mathcal{U}) \models \forall \bar{x}(\bar{x} \in X \leftrightarrow \phi(\bar{x}))\} .
\end{aligned}
$$

Thus $\cap \mathscr{K}$ is the intersection of all the structures belonging to $\mathscr{K}$ whereas $\operatorname{Def}(\mathscr{K})$ is the collection of all sets invariantly definable over $\mathscr{K}$.

If $T$ is a set of sentences of $L_{2}^{q_{l}}$ (i.e. a theory in $L_{2}^{q_{u}}$ ) then

$$
\operatorname{Mod}(T)=\{\mathscr{A}: \mathscr{A} \text { is a second order structure over } \mathcal{U} \text { and } \mathscr{A} \vDash T\}
$$

Definition 7. Let $\phi_{0}$ be a formula of $L_{2}^{Q_{2}} \cdot \operatorname{Ar}\left(\phi_{0}\right)$ is the smallest class of formulas of $L_{2}^{q u}$ such that

(i) $\phi_{0} \in \operatorname{Ar}\left(\phi_{0}\right)$,

(ii) every arithmetical formula of $L_{2}^{q_{l}}$ is in $\operatorname{Ar}\left(\phi_{0}\right)$,

(iii) if $\phi, \psi \in \operatorname{Ar}\left(\phi_{0}\right)$ then $\neg \phi, \phi \vee \psi, \exists x \phi \in \operatorname{Ar}\left(\phi_{0}\right)$.

A formula $\psi$ of $L_{2}^{q_{2}}$ is a $\Sigma_{1}^{1}\left(\phi_{0}\right)$ formula if it is of the form $\exists X_{1} \cdots \exists X_{n} \phi$ for some formula $\phi \in \operatorname{Ar}\left(\phi_{0}\right)$.

We call a relation $R(\bar{x}, \bar{X})\left(R \subset A^{n} \times \mathscr{P}\left(A^{n_{1}}\right) \times \cdots \times \mathscr{P}\left(A^{n_{k}}\right)\right.$ for some $\left.n, n_{1}, \cdots, n_{k}\right)$ arithmetical $\left(\Sigma_{1}^{1}\right)$ if for some arithmetical $\left(\Sigma_{1}^{1}\right)$ formula $\phi$ of $L_{2}^{q_{u}}$

$$
R=\{(\bar{x}, \bar{X}): \mathscr{P}(\mathcal{U}) \vDash \phi(\bar{x}, \bar{X})\} .
$$

A relation $R$ is $\Pi_{1}^{1}$ if $\neg R$ is $\Sigma_{1}^{1}$ and is $\Delta_{1}^{1}$ if both $R$ and $\neg R$ are $\Sigma_{1}^{1}$.

Recall that for $n \geqq 1, \mathscr{W}_{\mathscr{F}^{n}}=\left\{Y: Y\right.$ is well-founded on $\left.A^{n}\right\}$.

By $\Delta_{1}^{1}\left(\phi_{0}\right)$-Comp we mean the class of all the sentences of $L_{2}^{q}$ of the form

$$
\begin{gathered}
\forall \bar{Y}\left[\forall \bar{x}\left(\exists Z_{1} \phi\left(\bar{x}, Z_{1}, \bar{Y}\right) \leftrightarrow \forall Z_{2} \psi\left(\bar{x}, Z_{2}, \bar{Y}\right)\right)\right. \\
\left.\rightarrow \exists X \forall \bar{z}\left(\bar{z} \in X \leftrightarrow \exists Z_{1} \phi\left(\bar{z}, Z_{1}, \bar{Y}\right)\right)\right]
\end{gathered}
$$

where $X$ does not occur in $\phi$ and $\phi, \psi \in \operatorname{Ar}\left(\phi_{0}\right)$.

By $\Sigma_{1}^{1}\left(\phi_{0}\right)$-Comp we mean the class of all the sentences of $L_{2}^{q_{u}}$ of the form

$$
\forall \bar{Y} \exists X \forall \bar{z}(\bar{z} \in X \leftrightarrow \phi(\bar{z}, \bar{Y}))
$$

where $\phi \in \Sigma_{1}^{1}\left(\phi_{0}\right)$ and $X$ does not occur in $\phi$.

It is clear what we mean by $\Delta_{1}^{1}$-Comp, $\Sigma_{1}^{1}$-Comp or $\Sigma_{1}^{1}\left(\phi_{0}, W F(X)\right)$-Comp. 
Lemma 2. Let $\phi_{0}$ be a formula of $L_{2}^{u_{L}}$. For every $\Sigma_{1}^{1}\left(\phi_{0}\right)$ formula $\psi$ of $L_{2}^{u}$ there exists an $\operatorname{Ar}\left(\phi_{11}\right)$ formula $\phi$ such that if $\mathscr{A}$ is second order structure over $\mathcal{U}$ which satisfies the following two conditions:

$$
X, Y \in|\mathscr{A}| \Rightarrow X \times Y \in|\mathscr{A}|,
$$

(2) If $X \in|\mathscr{A}|$ and $X$ is a $l+m$-ary set $(l, m \geqq 1)$ then there exist sets $X_{1}, X_{2} \in|\mathscr{A}| l$-ary and $m$-ary respectively such that

$$
\begin{aligned}
& \mathscr{A} \vDash \forall \bar{z}\left(\bar{z} \in X_{1} \leftrightarrow \exists \bar{y}((\bar{z}, \bar{y}) \in X)\right), \\
& \mathscr{A} \vDash \forall \bar{y}\left(\bar{y} \in X_{2} \leftrightarrow \exists \bar{z}((\bar{z}, \bar{y}) \in X)\right),
\end{aligned}
$$

then

$$
\mathscr{A} \vDash \psi \leftrightarrow \exists X \phi .
$$

Proof. Assume for simplicity that $\psi$ is of the form $\exists X_{1} \exists X_{2} \psi_{1}\left(\bar{x}, X_{1}, X_{2}, \bar{X}\right)$ where $\psi_{1}\left(\bar{x}, X_{1}, X_{2}, \bar{X}\right)$ is a $\left(k, l, m, n_{1}, \cdots, n_{s}\right) \operatorname{Ar}\left(\phi_{0}\right)$-formula of $L_{2}^{u}$ for some $k, l, m, n_{1}, \cdots, n_{s}$ where $l, m \geqq 1$.

Let $\psi^{\prime}\left(\bar{x}, X, X_{2}, \bar{X}\right)$ be the following $\left(k, l+m, n_{1}, \cdots, n_{s}\right)$-formula of $L_{2}^{q}$ :

$$
\psi_{1 \bar{z}}\left(\bar{x}, \exists \bar{y}((\bar{z}, \bar{y}) \in X), X_{2}, \bar{X}\right)
$$

and let $\phi(\bar{x}, X, \bar{X})$ be the $\left(k, l+m, n_{1}, \cdots, n_{s}\right)$-formula of $L_{2}^{\mu}$ obtained from $\psi_{\bar{y}}^{\prime}(\bar{x}, X, \exists \bar{z}((\bar{z}, \bar{y}) \in Y), \bar{X})$ by replacing all the occurrences of $Y$ by $X$ (we have to make this small detour via $Y$ in order to avoid the clash of variables). Clearly $\phi \in \operatorname{Ar}\left(\phi_{0}\right)$.

Now it is easy to see that for all $\bar{x}$ and $\bar{X}$

$$
\mathscr{A} \vDash \exists X_{1} \exists X_{2} \psi_{1}\left(\bar{x}, X_{1}, X_{2}, \bar{X}\right) \leftrightarrow \exists X \phi(\bar{x}, X, \bar{X}) .
$$

Indeed, if for some $X_{1}$ and $X_{2} \mathscr{A} \vDash \psi_{1}\left(\bar{x}, X_{1}, X_{2}, \bar{X}\right)$ then $X_{1} \times X_{2} \in|\mathscr{A}|$ and clearly by Lemma $1 \mathscr{A} \vDash \phi(\bar{x}, X, \bar{X})$.

Conversely, if for some $X \mathscr{A} \vDash \phi(\bar{x}, X, \bar{X})$ then

$$
\begin{aligned}
& X_{1}=\{\bar{z}: \mathscr{A} \vDash \exists \bar{y}((\bar{z}, \bar{y}) \in X)\} \in|\mathscr{A}|, \\
& X_{2}=\{\bar{y}: \mathscr{A} \vDash \exists \bar{z}((\bar{z}, \bar{y}) \in X)\} \in|\mathscr{A}|
\end{aligned}
$$

and clearly by Lemma 1

$$
\mathscr{A} \vDash \psi_{1}\left(\bar{x}, X_{1}, X_{2}, \bar{X}\right) .
$$

Corollary 1. Let $\phi_{0}$ be a formula of $L_{2}^{u}$. Let $\mathscr{A}$ be a second order structure over $\mathcal{U}$. If $\mathscr{A} \vDash \Delta_{1}^{1}\left(\phi_{0}\right)$-Comp then $\mathscr{A}$ also satisfies the following scheme: 


$$
\forall \bar{Y}[\forall \bar{z}(\phi(\bar{z}, \bar{Y}) \leftrightarrow \neg \psi(\bar{z}, \bar{Y})) \rightarrow \exists X \forall \bar{z}(\bar{z} \in X \leftrightarrow \phi(\bar{z}, \bar{Y}))]
$$

where $X$ does not occur in $\phi$ and $\phi, \psi$ are $\Sigma_{1}^{1}\left(\phi_{0}\right)$ formulas.

Proof. By Lemma 2.

Suppose now that $\phi\left(x_{1}, \cdots, x_{n}, Y\right)$ is a $(n, n)$-formula of $L_{2}^{q^{\ell}}$. $\phi$ defines an operator $\Phi$ (not necessarily monotone) on the $n$-ary relations

$$
\Phi(S)=\left\{\left(x_{1}, \cdots, x_{n}\right): \mathscr{P}(\mathcal{U}) \vDash \phi\left(x_{1}, \cdots, x_{n}, S\right)\right\} .
$$

Define by induction

$$
\begin{gathered}
I_{\phi}^{\xi}=\bigcup_{\eta<\xi} I_{\phi}^{\eta} \cup \Phi\left(\bigcup_{\eta<\xi} I_{\phi}^{\eta}\right) \\
I_{\phi}=\bigcup_{\xi} I_{\phi}^{\xi}
\end{gathered}
$$

By the closure ordinal of $\phi,\|\phi\|$, we mean the least $\xi$ such that $I_{\phi}^{\xi}=\bigcup_{\eta<\xi} I_{\phi}^{\eta}$. $I_{\phi}$ is said to be inductively defined by $\phi$. Observe that

$$
I_{\phi}=I_{\phi}^{\|\phi\|}=\bigcup_{\eta<\|\phi\|} I_{\phi}^{\eta}
$$

By $|X|$ we denote the cardinality of a set $X$. By $\eta^{+}$we mean the least cardinal number greater than $\eta$.

If $\phi_{0}$ is a $(n, n)$-formula of $L_{2}^{\alpha_{u}}$ where $U=\left\langle A, R_{1}, \cdots, R_{n}\right\rangle$ then $\left\|\phi_{0}\right\|<|A|^{+}$. If $\bar{x} \in A^{n}$ then

$$
|\bar{x}|_{\phi_{0}}= \begin{cases}\text { least } \eta \text { such that } \bar{x} \in I_{\phi_{0}}^{\eta} & \text { if } \bar{x} \in I_{\phi_{0}} \\ |A|^{+} & \text {otherwise. }\end{cases}
$$

Observe that for every $\eta<|A|^{+}$there exists $X \subset A^{2}$ such that $\mathscr{P}(\mathcal{U}) \vDash W F(X)$ and $\|X\|=\eta$.

\section{§3. Nonmonotone inductive definitions and models of comprehension}

The first theorem we prove is the following:

THEOREM 1. Let $\phi_{0}$ be a $(1,1)$-formula of $L_{2}^{u}$. Let $\mathscr{A}$ be a second order structure over $\mathcal{U}$ such that $\mathscr{A} \vDash \Delta_{1}^{1}\left(\phi_{0}\right)$-Comp and $\phi_{0}$ is $\mathscr{A}$-absolute. Then

$$
\eta<h(\mathscr{A}) \Rightarrow I_{\phi_{0}}^{\eta} \in|\mathscr{A}| \text {. }
$$

Proof. Define a $(2,2,2)$-formula $\Phi(x, y, X, Y)$ of $L_{2}^{q u}$ as follows: 


$$
\Phi(x, y, X, Y) \leftrightarrow \exists z\left(z \in \operatorname{Fld}\left(X \Gamma_{x}\right) \wedge(z, y) \in Y\right)
$$

Let

$$
\psi(x, y, X, Y) \leftrightarrow \Phi(x, y, X, Y) \vee\left[\phi_{0 y}\left(y, \Phi\left(x, y^{\prime}, X, Y\right)\right) \wedge x \in \operatorname{Fld}(X)\right]
$$

(we avoid the clash of variables by appropriate renaming of the variables occurring in $\phi_{0}$ ).

Finally define

$$
\theta(X, Y) \leftrightarrow \forall x \forall y((x, y) \in Y \leftrightarrow \psi(x, y, X, Y)) .
$$

Observe that

$$
\psi(x, y, X, Y) \in \operatorname{Ar}\left(\phi_{0}\right)
$$

and

$$
\theta(X, Y) \in \operatorname{Ar}\left(\phi_{0}\right)
$$

so both are $\mathscr{A}$-absolute formulas.

We prove at first the following two facts:

(I) If $\mathscr{P}(U) \vDash W F(X)$ then for all $Y$

$$
\mathscr{P}(\mathscr{U}) \vDash \theta(X, Y) \Leftrightarrow Y=\left\{(x, y): x \in \operatorname{Fld}(X), y \in I_{\phi_{0}}^{\left\|X I_{x}\right\|}\right\} .
$$

(II) If $X \in|\mathscr{A}|$ and $\mathscr{P}(\mathscr{U}) \vDash W F(X)$ then

$$
\left\{(x, y): x \in \operatorname{Fld}(X), y \in I_{\phi_{0}}^{\left\|X I_{x}\right\|}\right\} \in|\mathscr{A}| .
$$

Suppose that $\mathscr{P}(\mathscr{U}) \vDash \mathrm{WF}(X)$. Let

$$
Y_{0}=\left\{(x, y): x \in \operatorname{Fld}(X), y \in I_{\phi_{0}}^{\left\|X I_{x}\right\|}\right\} .
$$

Proof of (I). We prove that $\mathscr{P}(\mathcal{U}) \vDash \theta\left(X, Y_{0}\right)$.

Let $x$ and $y$ be arbitrary. If $x \notin \operatorname{Fld}(X)$ then

$$
\mathscr{P}(\mathcal{U}) \vDash(x, y) \notin Y_{0} \wedge \neg \psi\left(x, y, X, Y_{0}\right) .
$$

So assume that $x \in \operatorname{Fld}(X)$. Then

$$
\begin{aligned}
\mathscr{P}(\mathcal{U}) \vDash(x, y) \in Y_{0} & \Leftrightarrow y \in I_{\phi_{0}}^{\left\|X I_{x}\right\|} \\
& \Leftrightarrow y \in \bigcup_{\eta<\left\|I_{x}\right\|} I_{\phi}^{\eta_{0}} \vee \phi_{0}\left(y, \bigcup_{\eta<\left\|x I_{x}\right\|} I_{\phi_{0}}^{\eta}\right)
\end{aligned}
$$

because 
$(z, x) \in X \Rightarrow\left(X \uparrow_{x} \uparrow_{z}=X \uparrow_{z}\right) \Leftrightarrow \exists z\left(z \in \operatorname{Fld}\left(X \uparrow_{x}\right) \wedge y \in I_{\phi_{0}}^{\left\|\left.X\right|_{z}\right\|}\right)$

$$
\vee \phi_{0}\left(y,\left\{y^{\prime}: \mathscr{P}(\mathcal{U}) \vDash \exists z\left(z \in \operatorname{Fld}\left(X \uparrow_{x}\right) \wedge y^{\prime} \in I_{\phi_{0}}^{\left\|X \Gamma_{z}\right\|}\right\}\right)\right.
$$

by Lemma 1

$$
\begin{aligned}
& \Leftrightarrow \mathscr{P}(\mathcal{U}) \vDash \Phi\left(x, y, X, Y_{0}\right) \vee \phi_{0 y}\left(y, \Phi\left(x, y^{\prime}, X, Y_{0}\right)\right) \\
& \Leftrightarrow \mathscr{P}(\mathcal{U}) \vDash \psi\left(x, y, X, Y_{0}\right) .
\end{aligned}
$$

Thus $\mathscr{P}(\mathcal{U}) \vDash \theta\left(X, Y_{0}\right)$.

Suppose now that for some $Y \mathscr{P}(\mathcal{U}) \vDash \theta(X, Y)$. Observe that then for all $x$ and $y$

$$
(x, y) \in Y \rightarrow x \in \operatorname{Fld}(X) .
$$

We prove by induction with respect to $\left\|X \Gamma_{x}\right\|$ that for all $x, y$

$$
(x, y) \in Y \leftrightarrow(x, y) \in Y_{0} .
$$

Let $x \in \operatorname{Fld}(X)$. Suppose that the claim is true for all pairs $(z, y)$ such that $\left\|X \uparrow_{z}\right\|<\left\|X \uparrow_{x}\right\|$. Then for all $y$

$$
\mathscr{P}(\mathcal{U}) \vDash(x, y) \in Y \Leftrightarrow \mathscr{P}(\mathcal{U}) \vDash \psi(x, y, X, Y)
$$

by Lemma 1

$$
\begin{gathered}
\Leftrightarrow \mathscr{P}(\mathcal{U}) \vDash \exists z\left(z \in \operatorname{Fld}\left(X \uparrow_{x}\right) \wedge(z, y) \in Y\right) \\
\vee \phi_{0}\left(y,\left\{y^{\prime}: \mathscr{P}(\mathcal{U}) \vDash \exists z\left(z \in \operatorname{Fld}\left(X \uparrow_{x}\right) \wedge(z, y) \in Y\right)\right\}\right)
\end{gathered}
$$

by induction hypotheses

$$
\begin{gathered}
\Leftrightarrow \mathscr{P}(\mathcal{U}) \vDash \exists z\left(z \in \operatorname{Fld}\left(X \uparrow_{x}\right) \wedge(z, y) \in Y_{0}\right) \\
\vee \phi_{0}\left(y,\left\{y^{\prime}: \mathscr{P}(\mathcal{U}) \vDash \exists z\left(z \in \operatorname{Fld}\left(X \uparrow_{x}\right) \wedge(z, y) \in Y_{0}\right)\right\}\right)
\end{gathered}
$$

by Lemma 1

$$
\Leftrightarrow \mathscr{P}(U) \vDash \psi\left(x, y, X, Y_{0}\right)
$$

by the above string of equivalences

$$
\Leftrightarrow \mathscr{P}(\mathcal{U}) \vDash(x, y) \in Y_{0} .
$$

Thus by induction $Y=Y_{0}$ which concludes the proof of (I).

Proof of (II). Assume additionally that $X \in|\mathscr{A}|$. We prove the claim by induction with respect to $\|X\|$. So suppose that the claim is true for $Z \in|\mathscr{A}|$ such that $\|Z\|<\|X\|$. Let for $x \in \operatorname{Fld}(X)$ 


$$
Y(x)=\left\{(z, y): z \in \operatorname{Fld}\left(\left.X\right|_{x}\right), y \in I_{\phi_{10}}^{\left\|X \Gamma_{x} \Gamma_{z}\right\|}\right\} .
$$

For $x \in \operatorname{Fld}(X)\left\|\left.X\right|_{x}\right\|<\|X\|$ and $\left.X\right|_{x} \in|\mathscr{A}|$ so by induction hypothesis and (I) combined with $\mathscr{A}$-absoluteness of $\theta$

$$
\forall x\left[x \in \operatorname{Fld}(X) \rightarrow\left(Y(x) \in|\mathscr{A}| \text { and } \mathscr{A} \vDash \theta\left(\left.X\right|_{x}, Y(x)\right)\right)\right] .
$$

We prove now that

$\mathscr{A} \vDash \forall x \forall y\left[\exists T \exists Y\left(\left.x \in \operatorname{Fld}(X) \wedge X\right|_{x}=T \wedge \theta(T, Y) \wedge \psi(x, y, X, Y)\right)\right.$

$$
\left.\leftrightarrow \forall T \forall Y\left(x \in \operatorname{Fld}(X) \wedge\left(\left(X \Gamma_{x}=T \wedge \theta(T, Y)\right) \rightarrow \psi(x, y, X, Y)\right)\right)\right] .
$$

Take arbitrary $x$ and $y$. Suppose that the left hand side of the equivalence holds. Then $x \in \operatorname{Fld}(X)$. By $\mathscr{A}$-absoluteness of $\theta$, (I) and (3)

$$
\mathscr{A} \vDash \psi(x, y, X, Y(x)) .
$$

Take now arbitrary $Y$ such that $\mathscr{A} \vDash \theta\left(\left.X\right|_{x}, Y\right)$. Then $\mathscr{P}(\mathscr{U}) \vDash \theta\left(\left.X\right|_{x}, Y\right)$, so by (I) $Y=Y(x)$. By the above $\mathscr{A} \vDash \psi(x, y, X, Y)$. Thus $\mathscr{A} \vDash \forall Y\left(\theta\left(\left.X\right|_{x}, Y\right) \rightarrow \psi(x, y, X, Y)\right)$, i.e. the right hand side of the equivalence holds.

Conversely, suppose that the right hand side holds. Then $x \in \operatorname{Fld}(X)$. By (3) $Y(x) \in|\mathscr{A}|$ and $\mathscr{A} \vDash \theta\left(\left.X\right|_{x}, Y(x)\right)$. Thus $\mathscr{A} \vDash \psi(x, y, X, Y(x))$. Hence $\mathscr{A} \vDash \exists Y\left(\theta\left(\left.X\right|_{x}, Y\right) \wedge \psi(x, y, X, Y)\right)$, i.e. the left hand side holds.

So (4) is proved. $\mathscr{A}$ satisfies $\Delta_{1}^{\prime}\left(\phi_{0}\right)$-Comp, so by (4) and Corollary 1

$$
\begin{array}{r}
\mathscr{A} \vDash \forall x \forall y\left[(x, y) \in Y_{1} \Leftrightarrow \exists T \exists Y(x \in \operatorname{Fld}(X) \wedge\right. \\
\left.\left.X \Gamma_{x}=T \wedge \theta(T, Y) \wedge \psi(x, y, X, Y)\right)\right]
\end{array}
$$

for some $Y_{1} \in|\mathscr{A}|$.

Thus for all $x$ and $y$

$$
\mathscr{A} \vDash(x, y) \in Y_{1} \Leftrightarrow \mathscr{A} \vDash x \in \operatorname{Fld}(X) \wedge \exists Y\left(\theta\left(\left.X\right|_{x}, Y\right) \wedge \psi(x, y, X, Y)\right)
$$

by (I) and (3)

$$
\Leftrightarrow \mathscr{A} \vDash x \in \operatorname{Fld}(X) \wedge \psi(x, y, X, Y(x))
$$

since $\psi$ is $\mathscr{A}$-absolute

$$
\Leftrightarrow \mathscr{P}(\mathscr{U}) \vDash x \in \operatorname{Fld}(X) \wedge \psi(x, y, X, Y(x))
$$

by the definition of $\psi$ and Lemma 1

$$
\begin{aligned}
& \Leftrightarrow x \in \operatorname{Fld}(X) \& \mathscr{P}(\mathscr{U}) \vDash \exists z\left(z \in \operatorname{Fld}\left(X \Gamma_{x}\right) \wedge y \in I_{\phi_{1}}^{\left\|x I_{x} r_{z}\right\|^{\prime}}\right) \\
& \vee \phi_{0}\left(y,\left\{y^{\prime}: \mathscr{P}(\mathcal{U}) \vDash \exists z\left(z \in \operatorname{Fld}\left(\left.X\right|_{x}\right) \wedge y^{\prime} \in I_{\phi_{01}}^{\left\|X I_{x} I_{z}\right\|}\right)\right\}\right)
\end{aligned}
$$


by the definition of $\left\|\left.X\right|_{x}\right\|$

$$
\begin{aligned}
& \Leftrightarrow x \in \operatorname{Fld}(X) \& \mathscr{P}(U) \vDash y \in \bigcup_{\eta<\left.|x|\right|_{x} \mid} I_{\phi_{n}}^{\eta} \\
& \vee \phi_{0}\left(y, \bigcup_{\eta<\| X I_{x}^{*} \mid} I_{\phi_{0}}^{\eta}\right) \\
& \Leftrightarrow x \in \operatorname{Fld}(X) \& y \in I_{\phi_{11}}^{\left\|X I_{x}\right\|} \text {. }
\end{aligned}
$$

Thus $Y_{1}=Y_{0}$. But $Y_{1} \in|\mathscr{A}|$ so the proof of (II) is concluded.

Let now $\eta<h(\mathscr{A})$. Then for some $X \in \mid \mathscr{A}$ such that $\mathscr{P}(\mathscr{U}) \vDash \operatorname{WF}(X)$ $\eta=\|X\|$. By (II)

$$
Y_{0}=\left\{(x, y): x \in \operatorname{Fld}(X), y \in I_{\phi_{0}}^{\left\|X I_{x}\right\|}\right\} \in|\mathscr{A}| .
$$

By comprehension there exists $Z \in|\mathscr{A}|$ such that

$$
\mathscr{A} \vDash \forall y\left(y \in Z \leftrightarrow \exists x\left((x, y) \in Y_{0}\right) \vee \phi_{0 y^{\prime}}\left(y^{\prime}, \exists x\left(\left(x, y^{\prime}\right) \in Y_{0}\right)\right)\right) .
$$

Observe that for all $y$

$$
\mathscr{A} \vDash y \in Z \Leftrightarrow \mathscr{P}(\mathcal{U}) \vDash \exists x\left((x, y) \in Y_{0}\right) \vee \phi_{0 y} .\left(y, \exists x\left(\left(x, y^{\prime}\right) \in Y_{0}\right)\right)
$$

by Lemma $1 \quad \Leftrightarrow y \in \bigcup_{\xi<i X \|} I_{\phi_{0}}^{\eta} \vee \mathscr{P}(\mathcal{U}) \vDash \phi_{11}\left(y, \bigcup_{\xi \in\|X\|} I_{\phi_{0}}^{\eta}\right)$

$$
\Leftrightarrow y \in I_{\phi_{\| \prime}}^{\|X\|} .
$$

Thus $Z=I_{\phi_{0}}^{\eta}$ which concludes the proof.

What are the conditions which, imposed on a structure $\mathscr{A}$, imply that $\left\|\phi_{0}\right\|<h(\mathscr{A})$ and $I_{\phi_{1}} \in|\mathscr{A}|$ ? Clearly we have to assume that $\mathscr{A}$ is a $\beta$-structure there are $\omega$-models of full comprehension which do not contain $\Pi_{1}^{1}-\Sigma_{1}^{1}$ sets (see e.g. Apt [1]), so in particular do not contain all sets inductively defined by arithmetical formulas.

Is it sufficient to assume that $\mathscr{A}$ is a $\beta$-model of $\Delta_{1}^{1}\left(\phi_{11}\right)$-Comp? The answer is no, because there exist acceptable structures on which every model of $\Delta_{1}^{1}$-Comp is a $\beta$-model.

We conjecture that $\Sigma_{1}^{1}\left(\phi_{0}\right)$-Comprehension is not sufficient either. Clearly one has to add the condition that $\phi_{0}$ is $\mathscr{A}$-absolute.

We prove the following theorem:

THEOREM 2. Let $\phi_{0}$ be a $(1,1)$-formula of $L_{2}^{\mu}$. Suppose that $\mathscr{A}$ is a $\beta$-structure over $\mathcal{U}$ which is a model of $\Sigma_{1}^{1}\left(\phi_{0}, \mathrm{WF}(X)\right)$-Comp and that $\phi_{0}$ is $\mathscr{A}$-absolute. Then $\left\|\phi_{0}\right\|<h(\mathscr{A})$. 
Proof. Let

$$
\chi(x, y) \Leftrightarrow \exists X \exists Y[\mathrm{WF}(X) \wedge \theta(X, Y) \wedge \exists z((z, x) \in Y \wedge(z, y) \notin Y)]
$$

where $\theta(X, Y)$ is the formula defined in the proof of Theorem 1. $\chi(x, y)$ is a $\Sigma_{1}^{\prime}\left(\phi_{0}, \mathrm{WF}(X)\right)$-formula, so there exists $Z \in|\mathscr{A}|$ such that for all $x$ and $y$

$$
\mathscr{A} \vDash(x, y) \in Z \Leftrightarrow \chi(x, y) \text {. }
$$

Observe that for all $x$ and $y$

$$
\begin{aligned}
(x, y) \in Z \Leftrightarrow & \mathscr{A} \vDash \mathrm{WF}(X) \wedge \theta(X, Y) \wedge \exists z((z, x) \in Y \wedge(z, y) \notin Y) \\
& \text { for some } X \in|\mathscr{A}| \text { and } Y \in|\mathscr{A}| \\
& \text { for some } X \in|\mathscr{A}| \text { and } Y \in|\mathscr{A}| \text { such that } \mathscr{P}(\mathscr{U}) \vDash \mathrm{WF}(X)
\end{aligned}
$$

because $\mathscr{A}$ is a $\beta$-structure and $\theta(X, Y)$ is $\mathscr{A}$-absolute

$$
\Leftrightarrow \mathscr{P}(\mathcal{U}) \vDash \theta(X, Y) \wedge \exists z((z, x) \in Y \wedge(z, y) \notin Y)
$$

for some $X \in|\mathscr{A}|$ such that $\mathscr{P}(\mathscr{U}) \vDash \mathrm{WF}(X)$

by (I) and (II)

$$
\begin{aligned}
& \Leftrightarrow \exists z\left(z \in \operatorname{Fld}(X) \wedge x \in I_{\phi_{0}}^{\left\|X \Gamma_{I^{2}}\right\|} \wedge y \notin I_{\phi_{0}}^{\left\|X I_{2}\right\|}\right) \\
& \Leftrightarrow|x|_{\phi_{0}}<h(\mathscr{A}) \&|x|_{\phi_{0}}<|y|_{\phi_{0}}
\end{aligned}
$$

because $h(\mathscr{A})$ is limit

$$
\Leftrightarrow x \in I_{\phi_{0}}^{\eta} \wedge y \notin I_{\phi_{0}}^{\eta} \text { for some } \eta<h(\mathscr{A}) .
$$

It follows that $Z$ is transitive and well-founded, i.e. $\mathscr{P}(\mathscr{U}) \vDash \mathrm{WF}(Z)$.

Suppose that $h(\mathscr{A}) \leqq\left\|\phi_{0}\right\|$. Then for every $\eta<h(\mathscr{A})$ there exists $x \in \operatorname{Fld}(Z)$ such that $|x|_{\phi_{0}}=\eta$. Clearly $\left\|\left.Z\right|_{x}\right\|=\eta$. Thus $h(\mathscr{A}) \leqq\|Z\|$, which is a contradiction.

THEOREM 3. Let $\phi_{0}$ be a $(1,1)$-formula of $L_{2}^{u}$, where $\mathcal{U}=\left\langle A, R_{1}, \cdots, R_{n}\right\rangle$. Let $\mathscr{l}\left(\phi_{1}\right)=\{\mathscr{A}: \mathscr{A}$ is a $\beta$-structure over $\mathcal{U}$, $\mathscr{A} \vDash \Sigma_{1}^{1}\left(\phi_{0}, \mathrm{WF}(X)\right)$-Comp and $\phi_{0}$ is $\mathscr{A}$-absolute $\}$.

Then

(i) $I_{\phi(c)} \in \cap \mathscr{K}\left(\phi_{0}\right)$.

(ii) $I_{\phi_{0}} \in \operatorname{Def}\left(\mathscr{K}\left(\phi_{0}\right)\right)$.

Moreover $I_{\phi_{0}}$ is invariantly definable over $\mathscr{K}\left(\phi_{0}\right)$ by a $\Sigma_{1}^{1}\left(\phi_{0}, \mathrm{WF}(X)\right)$-formula. 
(iii) There exists a $\Sigma_{1}^{1}\left(\phi_{0}, \mathrm{WF}(X)\right)$-formula $\psi_{0}(x, y)$ such that

(i') $\psi_{0}(x, y)$ is $\mathscr{A}$-absolute for $\mathscr{A} \in \mathscr{E}\left(\phi_{11}\right)$,

(ii') $\left\|Z_{0}\right\|=\left\|\phi_{0}\right\|$ where $Z_{0}=\left\{(x, y): \mathscr{P}(\mathscr{U}) \vDash \psi_{0}(x, y)\right\}$.

Proof of (i). By Theorems 1 and 2 .

Proof of (ii). Observe that for all $x$

$$
\mathscr{P}(U) \vDash x \in I_{\phi_{1}} \leftrightarrow \exists X \exists Y[\mathrm{WF}(X) \wedge \theta(X, Y) \wedge \exists z((z, x) \in Y)] .
$$

Indeed,

$$
\begin{aligned}
x \in I_{\phi_{1}} & \Leftrightarrow x \in I_{\phi_{11}}^{\eta+1} \text { for some } \eta<|A|^{+} \\
& \Leftrightarrow x \in I_{\phi_{11}}^{\left\|X \Gamma_{z}\right\|} \text { for some } X \text { such that } W F(X) \text { and } z \in \operatorname{Fld}(X)
\end{aligned}
$$

by (I)

$$
\Leftrightarrow \exists X \exists Y[W F(X) \wedge \theta(X, Y) \wedge \exists z((z, x) \in Y)] .
$$

It remains to prove that the formula

$$
\exists X \exists Y[\mathrm{WF}(X) \wedge \theta(X, Y) \wedge \exists z((z, x) \in Y)]
$$

is $\mathscr{A}$-absolute for $\mathscr{A} \in \mathscr{K}\left(\phi_{0}\right)$.

Let $\mathscr{A} \in \mathscr{K}\left(\phi_{0}\right)$. By Theorem 2 there exists $Z \in|\mathscr{A}|$ such that $\mathscr{P}(\mathscr{U}) \vDash \mathrm{WF}(Z)$ and $\|Z\|=\left\|\phi_{0}\right\|$.

We have for all $x$

$$
\begin{aligned}
\mathscr{P}(\mathcal{U}) \vDash & \exists \exists Y[\mathrm{WF}(X) \wedge \theta(X, Y) \wedge \exists z((z, x) \in Y)] \\
& \Rightarrow x \in I_{\phi_{1}} \\
& \Rightarrow x \in I_{\phi_{0}}^{\eta} \text { for some } \eta<\|Z\| \\
& \Rightarrow x \in I_{\phi_{0}}^{\left\|Z I_{z}\right\|} \text { for some } z \in \mathrm{Fld}(Z) \\
& \Rightarrow \mathscr{P}(\mathcal{U}) \vDash \exists Y[\theta(Z, Y) \wedge \exists z((z, x) \in Y)] \\
& \Rightarrow \mathscr{A} \vDash \exists Y[\theta(Z, Y) \wedge \exists z((z, x) \in Y)] \\
& \Rightarrow \mathscr{A} \vDash \exists X \exists Y[\mathrm{WF}(X) \wedge \theta(X, Y) \wedge \exists z((z, x) \in Y)] .
\end{aligned}
$$

by (I)

by (II)

Implication in the other direction follows from the fact that $W F(X)$ and $\theta(X, Y)$ are $\mathscr{A}$-absolute formulas.

Proof OF (iii). Let

$$
\begin{array}{r}
\psi_{0}(x, y) \leftrightarrow \exists X \exists Y[\mathrm{WF}(X) \wedge \theta(X, Y) \wedge \exists t((t, y) \in Y \\
\wedge \exists z((z, x) \in Y \wedge(z, y) \notin Y)]
\end{array}
$$


and let $Z_{01}=\left\{(x, y): \mathscr{P}(\mathscr{U}) \vDash \psi_{0}(x, y)\right\}$. Then for all $x$ and $y$

$$
\mathscr{P}(\mathscr{U}) \vDash(x, y) \in Z_{0} \Leftrightarrow y \in I_{\phi_{1}} \&|x|_{\phi_{10}}<|y|_{\phi_{1}}
$$

as a string of equivalences analogous to that in the proof of Theorem 2 shows (in place of $\mathscr{A}$ we should take $\mathscr{P}(\mathscr{U})$ ).

The proof that $\psi_{0}(x, y)$ is $\mathscr{A}$-absolute for $\mathscr{A} \in \mathscr{K}\left(\phi_{0}\right)$ is analogous to the appropriate proof from part (ii).

Let $\operatorname{WF}^{n}(X)$ be the following $(0,2 n)$-formula of $L_{2}^{\prime \prime}$ where $U=$ $\left\langle A, R_{1}, \cdots, R_{n}\right\rangle: \mathrm{WF}^{n}(X) \leftrightarrow X$ is a well-founded transitive relation on $A^{n}$.

Clearly all the results of this section hold for $(n, n)$-formulas instead of $(1,1)$-ones after replacing throughout the section each occurrence of the formula WF $(X)$ (including the definition of $h(\mathscr{A})$ - we denote this changed ordinal by $\left.h^{n}(\mathscr{A})\right)$ by $\mathrm{WF}^{n}(X)$.

From the proofs of Theorems 1 and 2 we can easily extract

THEOREM 4. Suppose that for some $n \geqq 1$ the relation $\mathscr{W}^{n} \mathscr{F}^{n}$ is arithmetical on U. Then

$$
\left\{I_{\phi_{1}}: \phi_{0} \text { is a }(n, n) \text {-arithmetical formula of } L_{2}^{\prime \prime}\right\} \subset \cap \operatorname{Mod}\left(\Sigma_{1}^{1}\right. \text {-Comp). }
$$

Proof. For some arithmetical formula $\psi$ of $L_{2}^{\prime \prime}$

$$
\mathscr{P}(\mathscr{U}) \vDash \mathrm{WF}^{n}(X) \leftrightarrow \psi(X) .
$$

Suppose that $\phi_{01}$ is a $(n, n)$-arithmetical formula of $L_{2}^{\prime \prime}$. Then $\Delta_{1}^{\prime}\left(\phi_{10}\right)$-Comp $=$ $\Delta$-Comp. Let $\mathscr{A}$ be a second order structure over $U$ which is a model of $\sum_{1}^{1}$-Comp

Let $\chi(\bar{x}, \bar{y})$ be the following $(2 n)$-formula of $L_{2}^{\prime \prime}$

$$
\chi(\bar{x}, \bar{y}) \leftrightarrow \exists X \exists Y[\psi(X) \wedge \theta(X, Y) \wedge \exists \bar{z}((\bar{z}, \bar{x}) \in Y \wedge(\bar{z}, \bar{y}) \notin Y)]
$$

where $\theta(X, Y)$ is the appropriate $(0,2 n, 2 n)$-formula defined in the proof of Theorem 1 .

$\chi(\bar{x}, \bar{y})$ is a $\Sigma_{1}^{1}$ formula, so there exists $Z \in \mid \mathscr{A}$ such that

$$
\mathscr{A} \vDash \forall \bar{x} \forall \bar{y}((\bar{x}, \bar{y}) \in Z \leftrightarrow \chi(\bar{x}, \bar{y})) .
$$

The same string of equivalences like in the proof of Theorem 2 shows that for all $\bar{x}$ and $\bar{y}$

$$
\left.(\bar{x}, \bar{y}) \in Z \Leftrightarrow \bar{x}\right|_{\phi_{1}}<h^{n}(\mathscr{A}) \&|\bar{x}|_{\phi_{1}}<|\bar{y}|_{\phi_{11}}
$$

which analogously implies that $\left\|\phi_{0}\right\|<h(\mathscr{A})$. Due to Theorem $1 I_{\phi_{1}} \in \mid \mathscr{A}$. 
COROLlary 2. Barwise-Grilliot theorem (see Moschovakis [2] p. 140) is false if one omits the assumption of countability.

Proof. There are acceptable structures $\mathscr{U}$ on which all the relations $\mathrm{WF}^{n}$ are arithmetical (see Moschovakis [2] theorem 6C.3). By Theorem 4 the intersection of all models of $\Sigma_{1}^{1}$-Comp contains all inductive relations on $\mathcal{U}$, so by corollary 5D.3 from Moschovakis [2] it is different from the family of all hyperarithmetical relations on $U$.

Note. The other way to prove the above corollary is to use theorem 7A.1 from Moschovakis [2] in connection with theorem 7F.1. The advantage of the proof presented above is that it is much more elementary - it doesn't even use any of two Stage Comparison Theorems.

\section{$\S 4$. Invariant definability over models of $\Delta_{1}^{1}$-Comprehension}

Throughout this section we shall use freely several results from Moschovakis [2]. Instead of writing e.g. "by theorem 7A.2 from Moschovakis [2]" we shall simply write "by theorem 7A.2".

All the previously unexplained notations can be found in Moschovakis [2].

THEOREM 5. Let $U$ be an acceptable structure. Let $T$ be an inductive theory in

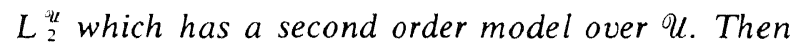

$$
\operatorname{Def}(\operatorname{Mod}(T)) \subset \Delta_{1}^{1} \text {. }
$$

Here, of course, $\Delta_{1}^{1}$ denotes the family of all $\Delta_{1}^{1}$ first order relations on $\mathcal{U}$.

Proof. Let $X \in \operatorname{Def}(\operatorname{Mod}(T))$. For some formula $\phi$ of $L_{2}^{\mathscr{Q}}$ which is $\mathscr{A}$ absolute for $\mathscr{A} \in \operatorname{Mod}(T)$

$$
\mathscr{P}(\mathcal{U}) \vDash \forall \bar{x}(\bar{x} \in X \leftrightarrow \phi(\bar{x})) .
$$

Thus for all $\bar{x}=\left(x_{1}, \cdots, x_{n}\right)$

$$
\begin{aligned}
\bar{x} \in X & \Leftrightarrow \mathscr{P}(\mathscr{U}) \vDash \phi(\bar{x}) \\
& \Leftrightarrow \text { for every } \mathscr{A} \in \operatorname{Mod}(T) \mathscr{A} \vDash \phi(\bar{x}) \\
& \Leftrightarrow \forall Z\left[\operatorname{Mod}^{T}(Z) \rightarrow \operatorname{Sat}_{2}\left({ }^{\prime} \phi^{\prime}, Z, \phi,\left\langle x_{1}, \cdots, x_{n}\right\rangle\right)\right]
\end{aligned}
$$

(we are using here the notation of lemma 7E.2 and theorem 8 C.2). $\operatorname{Mod}^{T}(Z)$ is a $\Sigma_{1}^{1}$ relation (see theorem $8 C .2$ ) and $\operatorname{Sat}_{2}(a, Z, Y, b)$ is a $\Delta_{1}^{1}$ relation (see lemma 7E.2 and theorem 6B.5). Thus $X$ is a $\Pi_{1}^{1}$ relation. Also $\neg X \in \operatorname{Def}(\operatorname{Mod}(T))$, so $\neg X$ is a $\Pi_{1}^{1}$ relation relation, as well. Thus $X$ is $\Delta_{1}^{1}$. 
THEOREM 6. Let $U$ be an acceptable structure. Let $T$ be a theory in $L_{2}^{u}$ such that $\Delta_{1}^{1}$-Comp $\subset T$ and $T$ has a second order model over $U$. Then

$$
\mathscr{H} \mathscr{E}(\mathscr{U}) \subset \operatorname{Def}(\operatorname{Mod}(T)) \text {. }
$$

Proof. Suppose that $X_{0} \in \mathscr{H} \mathscr{E}(\mathcal{U})$. By theorem 7D.1 there exists a formula $\phi(\bar{x}, X)$ of $L^{u}$ such that for all $\bar{x}$

$$
\bar{x} \in X_{0} \Leftrightarrow \exists X \phi(\bar{x}, X) \Leftrightarrow \exists X(X \in \mathscr{H} \mathscr{E}(\mathcal{U}) \& \phi(\bar{x}, X)) .
$$

Let $\mathscr{A} \in \operatorname{Mod}(T)$. Then $\mathscr{H} \mathscr{E}(\mathcal{U}) \subset|\mathscr{A}|$ by theorem $7 \mathrm{E} .1$.

We have for all $\bar{x}$

$$
\begin{aligned}
\mathscr{P}(U) \vDash \exists X \phi(\bar{x}, X) & \Rightarrow \mathscr{P}(\mathscr{U}) \vDash \phi(\bar{x}, X) \text { for some } X \in \mathscr{H} \mathscr{E}(U) \\
& \Rightarrow \mathscr{P}(\mathscr{U}) \vDash \phi(\bar{x}, X) \text { for some } X \in|\mathscr{A}| \\
& \Rightarrow \mathscr{A} \vDash \exists X \phi(\bar{x}, X) .
\end{aligned}
$$

Thus $\exists X \phi(\bar{x}, X)$ is $\mathscr{A}$-absolute which shows that $X_{0} \in \operatorname{Def}(\operatorname{Mod}(T))$.

Combining these two theorems we get

Corollary 3. Let $U$ be a countable acceptable structure. Let $T$ be an inductive theory in $L_{2}^{u}$ such that $\Delta_{1}^{1}$-Comp $\subset T$ and $T$ has a second order model over $U$. Then

$$
\mathscr{H} \mathscr{E}(\mathscr{U})=\operatorname{Def}(\operatorname{Mod}(T)) .
$$

Proof. By theorem 8A.1 $\mathscr{H} \mathscr{E}(\mathcal{U})=\Delta_{1}^{1}$, so the claim follows from Theorems 5 and 6.

The above statement is not true for uncountable acceptable structures. It follows from the following theorem.

THEOREM 7. Let $\mathcal{U}$ be an acceptable structure. Suppose that for some $n \geqq 1$ $\mathscr{W F}^{n}$ is arithmetical on $\mathcal{U}$. Then

$$
\left\{I_{\phi_{0}}: \phi_{0} \text { is a }(n, n) X \text {-positive formula of } L^{u}\right\} \subset \operatorname{Def}\left(\operatorname{Mod}\left(\Delta_{1}^{1}-\operatorname{Comp}\right)\right) \text {. }
$$

Proof. Let $\phi_{0}$ be a $(n, n) X$-positive formula of $L^{q}$ and let $\mathscr{A}$ be a model of $\Delta_{1}^{\prime}$-Comp.

By theorem 7A.1 there exists a formula $\phi(\bar{x}, Y)$ of $L^{4}$ such that for all $\bar{x}$

$$
\begin{aligned}
\bar{x} \in I_{\phi_{1}} & \Leftrightarrow \exists Y\left[\mathscr{W}_{\mathscr{F}^{n}}(Y) \& \phi(\bar{x}, Y)\right] \\
& \Leftrightarrow \exists Y\left[Y \in \mathscr{H} \mathscr{E}(U) \& \mathscr{W} \mathscr{F}^{n}(Y) \& \phi(\bar{x}, Y)\right] .
\end{aligned}
$$

For some formula $\psi$ of $L^{u}$ 


$$
\mathscr{W}_{\mathscr{F}^{n}}(X) \Leftrightarrow \mathscr{P}(\mathcal{U}) \vDash \psi(X)
$$

for all $X \subset A^{2 n}$. Then

$$
\mathscr{P}(\mathcal{U}) \vDash \forall \bar{x}\left(\bar{x} \in I_{\phi_{0}} \leftrightarrow \exists Y[\psi(Y) \wedge \phi(\bar{x}, Y)]\right) .
$$

We have for all $\bar{x}$

$$
\begin{array}{ll}
\qquad \mathscr{P}(\mathcal{U}) \vDash \exists Y[\psi(Y) \wedge \phi(\bar{x}, Y)] \Rightarrow & \mathscr{P}(\mathcal{U}) \vDash \psi(Y) \wedge \phi(\bar{x}, Y) \\
& \text { for some } Y \in \mathscr{H} \mathscr{E}(\mathcal{U}) \\
\text { by theorem 7F.1 } & \Rightarrow \mathscr{P}(\mathcal{U}) \vDash \psi(Y) \wedge \phi(\bar{x}, Y) \\
& \text { for some } Y \in|\mathscr{A}| \\
& \Rightarrow \mathscr{A} \vDash \exists Y[\psi(Y) \wedge \phi(\bar{x}, Y)]
\end{array}
$$

which shows that $\exists Y[\psi(Y) \wedge \theta(Y, \bar{x})]$ is $\mathscr{A}$-absolute. Thus $I_{\phi_{0}} \in$ $\operatorname{Def}\left(\operatorname{Mod}\left(\Delta_{1}^{1}-\operatorname{Comp}\right)\right)$.

We are unable to characterize the sets which belong to $\operatorname{Def}\left(\operatorname{Mod}\left(\Delta_{1}^{1}-\operatorname{Comp}\right)\right)$ in the case of arbitrary acceptable structures. Observe, however, that the following theorem holds.

THEOREM 8. Let $U=\left\langle A, R_{1}, \cdots, R_{n}\right\rangle$ be an acceptable structure. There exists an inductive set $I$ such that

$$
\operatorname{Def}\left(\operatorname{Mod}\left(\Delta_{1}^{1}-\operatorname{Comp}\right)\right) \subset \mathscr{H} \mathscr{E}(\mathscr{U}, I)
$$

Proof. Let $I$ be an inductive binary relation which parametizes the unary inductive relations (see theorem 5D.2). Let $X \in \operatorname{Def}\left(\operatorname{Mod}\left(\Delta_{1}^{1}-\operatorname{Comp}\right)\right)$. Then for all $x_{1}, \cdots, x_{r}$

$$
\mathscr{P}(\mathcal{U}) \vDash\left(x_{1}, \cdots, x_{r}\right) \in X \Leftrightarrow\left\langle A, \mathscr{H} \mathscr{E}(\mathcal{U}), R_{1}, \cdots, R_{n}\right\rangle \vDash \phi\left(x_{1}, \cdots, x_{r}\right) .
$$

Let $k$ be the highest arity of second order variables occurring in $\phi$. Then clearly for all $x_{1}, \cdots, x_{r}$

$$
\mathscr{P}(\mathcal{U}) \vDash\left(x_{1}, \cdots, x_{r}\right) \in X \Leftrightarrow\left\langle A, \bigcup_{m \leqq k} \mathscr{H}_{\mathscr{E}^{m}}(\mathcal{U}), R_{1}, \cdots, R_{n}\right\rangle \vDash \phi\left(x_{1}, \cdots, x_{r}\right) .
$$

By theorem 5D.4 for every $l$ there exists a $(l+1)$-ary inductive set $I^{\prime}$ which parametrizes $\mathscr{H}_{\mathscr{E}^{\prime}}(\mathcal{U})$.

Let $J=\left\{\langle\underline{m}, a\rangle: a \in I^{m}, m \leqq k\right\}$ (here $\underline{m}$ is the $m$-th integer in the copy of $\omega$ in a coding scheme fixed throughout the proof). Clearly $J$ is inductive.

By lemma 7E.2 for all $x_{1}, \cdots, x_{r}$ 


$$
\mathscr{P}(\mathscr{U}) \vDash\left(x_{1}, \cdots, x_{r}\right) \in X \Leftrightarrow \operatorname{Sat}_{2}\left(\phi^{\prime}, J, \phi,\left\langle x_{1}, \cdots, x_{r}\right\rangle\right)
$$

where $\mathrm{Sat}_{2}$ is a second order hyperelementary relation.

This easily implies that $X$ is hyperelementary on $(\mathcal{U}, J)$. For some $a$ $J=\{x:(a, x) \in I\}$ thus $X \in \mathscr{H} \mathscr{E}(\mathcal{U}, I)$.

It seems likely that the inclusion in the above theorem could be replaced by equality. We are, however, unable to prove it.

\section{REFERENCES}

1. K. R. Apt, $\omega$-models in analytical hierarchy, Bull. Acad. Polon. Sci. 20 (1972), 901-904.

2. Y. N. Moschovakis, Elementary Induction on Abstract Structures, North-Holland, Amsterdam, 1974 .

3. Y. N. Moschovakis, On monotone inductive definability, Fund. Math. 82 (1974), 39-83.

\section{Mathematical Centre}

2E BoerhaAvestraat 49

AMSTERDAM 1005, THE NETHERLANDS 